\section{Ueber im Westen beobachtetes sogen. Fünftagefieber.}

Von Prof. Dr. 0. Frese,

z. Z. Stabsarzt bei einem Feldlazarett im Westen.

Eine bisher unbekannte ,Kriegskrankheit", mit periodischen Fieberanfällen in meist fünftägigem Abstand, ist in vergangenem und diesem Jahr beim Ostheer in ziemlich zahlreichen Fällen beobachtet worden. trennt lagen. In zwei Fällen ist die Erkrankung im Lazarett selbst aufgetreten, wo sich der eine wegen leichter nervöser Herzbeschwerden, der andere wegen eines unbedeutenden Ekzems seit 12 bezw. 5 Tagen fieberfrei aufhielten. Auch $\mathrm{His}$ hat dies bei seinem Material mehrere Male beobachtet. Außer neun charakteristischen Fällen habe ich eine Anzahl zweifelhafter gesehen, bei denen die Beobachtung zum Teil noch nicht abgeschlossen ist und die ich deshalb hier nicht berücksichtigen will.

Bei einigen Kranken entsprach die Fieberkurve genau dem Fünftagetypus, so z. B. bei Musketier M. (siehe Kurve 1), annähernd auch bei Gefreiten Gr. (siehe Kurve 2): Bei diesem Kranken ist die geringe Höhe einzelner Anfälle auf die verabreichten Medikamente zu beziehen. Manchmal war das fieberfreie Intervall kürzer, sodaß der neue Fieberanstieg schon am dritten oder vierten Tage erfolgte; auch bei den einzelnen Kranken wechselte das zuweilen (siehe Kurve 3 und 4). Einmal sah ich den neuen Anfall erst am sechsten Tage eintreten. Alle Autoren stimmen darin überein, daß der Fünftageabstand zwar der häufigste ist, aber nicht immer genau eingehalten wird. Bis zu sieben Anfälle bei einem Patienten habe ich beobachtet; meist waren es 4-5, doch hatten wahrscheinlich zum Teil vor der Aufnahme schon einige Anfälle stattgefunden.

Die Krankheit begann ohne wesentliche Prodrome mit leichtem Frost. Ein eigentlicher Schüttelfrost, wie bei Malaria, wurde nicht beobachtet. Einzelne Kranke spürten nur ein leichtes Frösteln oder überhaupt nichts derartiges, besonders bei den späteren Anfällen.
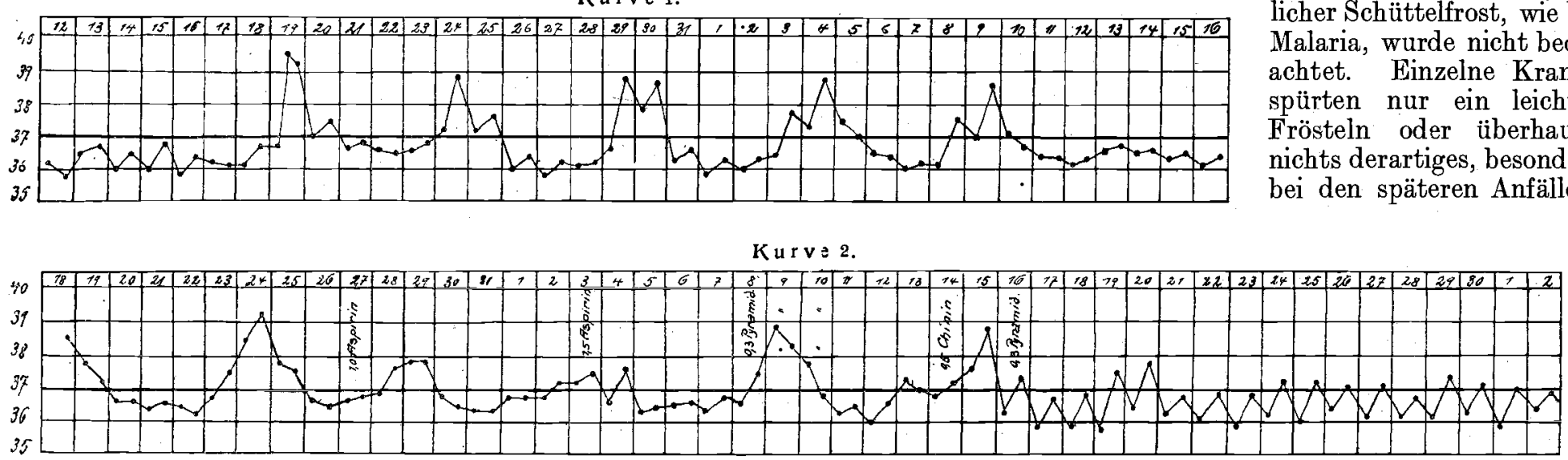

Kurve 3 .

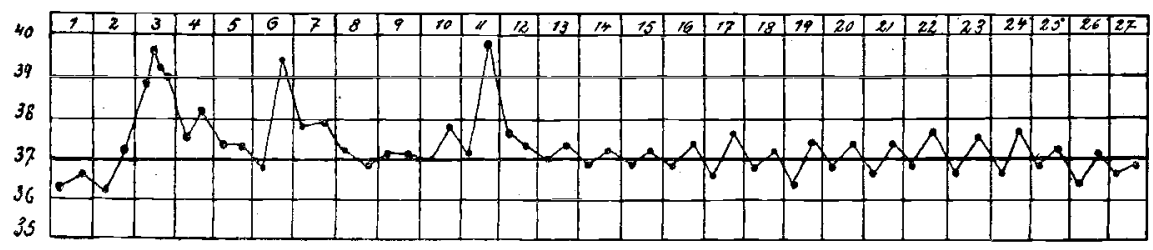

Kurve 4.J

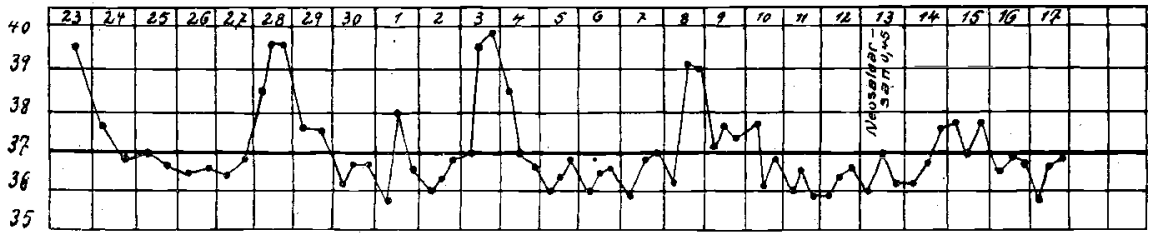

Es hängt dies wohl damitzusammen, daß das Fieber langsam anstieg," wie ich bei allen Fällen feststellen konnte, die ein- oder zweistündlich - auch nachts - gemessen worden sind. Ich möchte dies besonders hervorheben, weil andere Autoren von ,rascher Temperatursteigerung " sprechen. Die Betrachtung der Kurven 5 und 6 läßt diese Verhältnisse deutlich erkennen. Bis zur Erreichung des ersten Temperaturgipfels - von der $37^{\circ}$-Linie an gerechnet - vergehen z. B. bei Sch. (Kurve 5) 8 Stunden, bei Oe. (Kurve 6) 12-14 Stunden. Dann folgt ein Fastigium von mehrstündiger Dauer (8-20 Stunden), das mehrere kleine Senkungen und Erhebungen erkennen läßt, sodaß sich meist zwei oder drei kleine Zacken ausprägen. Der Fieberabfall erfolgt ebenfalls langsam; ungefähr in demselben Tempo wie der Anstieg.

Es haben darüber berichtet: $\mathrm{Hi}^{2}$ ), $\operatorname{Korbsch}^{3}$ ), Werner ${ }^{4}$ ), Brasch ${ }^{5}$ ), Hasenbelg ${ }^{5}$ ) und Rumpel ${ }^{6}$ ).

Soviel mir bekannt, sind solche Fälle beim Westheer bisher nicht beobachtet bezw. veröffentlicht worden. ${ }^{7}$ ) Ich will deshalb kurz über mehrere derartige Erkrarkungen berichten, die in einem Feldlazarett im Norden Frankreichs vom März bis Juli d. J. zu meiner Beobachtung kamen. Die meisten der Erkrankten waren überhaupt niemals im Osten gewesen; sie verteilten sich auf verschiedene Formationen, die örtlich ge-

1) Nach R.Paltauf im Handb. von Krehl-Marchand, daselbst übrige Literatur.

2) B. kl. W. 1916 Nr. 12 u. 27. - 3) D. m. W. 1916 Nr. 12 4) M. m. W. 1916 Nr. 11. - $^{5}$ ) M. m. W. 1916 Nr. 23. - $^{6}$ ) D. m. W. 1916 Nr. 22. - $\left.{ }^{7}\right)$ Wie ich nachträglich höre, soll sich auch die Veröffentlichun von Korbsch auf Beohachtungen aus dem Westen stützen. Aus der Arbeit selbst geht dies nicht hervor.
Mit ihm war meist ein mäßiger Schweißaustritt verbunden, doch betrug in den genau gemessenen Fällen zwischen 28 und 34 Stunden. Die absolute Höhe der Gipfeltemperatur war niemals beträchtlich; sie lag selten erheblich über $39^{\circ}$, öfters hielt sie sich unter diesem Wert, namentlich bei den späteren Anfällen. Der Beginn des Fiebers fiel bei den Patienten, wo die Messungen oft genug vorgenommen sind, um ein sicheres Urteil zu gestatten, bei allen Anfällen fast ge na u a uf dieselbe Stunde; so begann dasselbe z. B. bei einem Kranken stets um 5 Uhr nachmittags, bei einem anderen zwischen 3 und 5 Uhr vormittags. An Kurven, welche nur die Abend- und Morgentemperatur wiedergeben, traten diese Eigenarten natürlich nicht deutlich hervor. Die Kurve sieht dann viel steilschenkliger aus, und ein Teil der charakteristischen Zacken fällt hinweg, zumal wenn während der Nachtstunden die 
Messung ausgesetzt wird. Hieraus erklärt sich vielleicht die oben erwähnte Annahme eines raschen Temperaturanstiegs seitens anderer Autoren. Die Temperaturkurve des Fünftagefiebers verhält sich also nach meinen Beobachtungen ganz anders als die der sonst bei uns vorkommenden periodischen Fieber. Bei der Malaria tertiana, die wir hier sehr häufig beobachten, steigt die Temperatur innerhalb von 2-4 Stunden auf ihren höchsten Wert, um ohne länger dauerndes Fastigium verhältnismäßig langsam abzusinken (in 6-8, zuweilen bis zu 20 Stunden). Die Basis des ganzen Anfalls ist meist ziemlich schmal. Eine Aehnlichkeit mit dem Pünftagefieber besteht in

sucht wurde. Hasenbalg vermißte sie ebenfalls bei seinen Kranken, während die übrigen, oben genannten Autoren sie meist vorfanden. In einem Fall waren anfangs geringe Durchfälle vorhanden, einmal wurde ein Herpes labialis notiert. Der Urin war stets eiweißfrei. Bei Musketier Sch. wurde dreimal das Auftreten von Roseolen beobachtet, was sonst nur von Brasch bei seinen Kranken gesehen wurde. Sie zeigten sich am Schluß des Fieberanfalls, um am ersten Intervalltage noch an Zahl zuzunehmen. Sie fanden sich namentlich in der Oberbauchgegend und den unteren seitlichen Teilen des Brustkorbs, einmal in geringer Zahl auch an den Armen. Es wurden bei einem Anfall bis zu 50 Stück gezählt. Sie waren rundlich, etwa linsengroß, hellrot und verschwanden völlig auf Fingerdruck; die Haut fühlte sich nicht infiltriert an. Nach etwa 24 Stunden waren sie bereits stark abgeblaßt, nach 48 Stunden verschwunden. Bei vier Kranken habe ich genaue und

der Zackenbildung nahe dem Fastigium, die bei stündlicher Messung auch bei Malaria häufig nachweisbar ist.

Der Rekurrensfieberanfall, über den ich eigene Erfahrungen nicht besitze, unterscheidet sich vom Fünftagefieber durch den viel steileren An- und Abstieg der Temperatur, die höheren Maxima und das langanhaltende Fastigium; ähnlich sind wieder die Zackenbildungen auf der Höhe des Fiebers.

Diese Eigentümlichkeiten der verschiedenen Fieberkurven wird man beim ersten Anfall eines Fünftagefiebers differential diagnostisch verwerten können.

häufige Blutuntersuchungen vorgenommen. Das Blut wurde meist während des Fieberanstiegs oder auf der Höhe desselben entnommen. Es wurden zahlreiche frische und gefärbte.Präparate untersucht, letztere in dünnem Ausstrich und nach der Dicketropfenmethode; unter anderem wurde auch das Roseolenblut in dieser Weise untersucht (Giemsa-Färbung). Parasiten irgendwelcher Art konnte ich niemals nachweisen. Das Aussehen der Erythrozyten war normal. Basophile Tüpfelung war nicht vorhanden. Die Leukozyten waren vermehrt.

Brasch fand in seinen Fällen manchmal diplokokkenartige Gebilde im Blut, die aber nicht immer völlig eindeutig waren; His sah konstant im Blute Kokken oder Diplokokken, die auch in Stäbchenform auftraten und Entwicklungsstadien eines Protozoon sein sollen. Korbsch berichtet von $7-10 \mu$ langen, gestreckten oder gewundenen Fädchen, die er in der Mehrzahl der Fälle bei Giemsa-Färbung nachweisen konnte. $\mathrm{Knack}^{1}$ ) hält diese Gebilde übrigens für Degenerationsprodukte roter Blutkörperchen. Auch Wer$n e r^{2}$ ) hat in einem Fall eine Spirochäte ge-

Im Intervall hielt sich die Temperatur in unseren Fällen meist zwischen $36^{\circ}$ und $37^{\circ}$, ohne auf so niedrige Werte abzusinken, wie wir dies häufig bei Malaria sehen. Zuweilen ist zwischen den Anfällen bei genauer Messung eine kurzdauernde kleine Temperaturerhöhung festzustellen (siehe Kurve 7). Die späteren Anfälle gestalten sich manchmal un regelmäßig, worauf auch schon andere Beobachter hingewiesen haben. So schließt sich z. B. an den vierten Anfall des Kan. Oe. nochmals sofort eine leichte Temperatursteigerung von $37-38^{\circ}$ an, die fast 20 Stunden anhält (siehe Kurve 6 und 7), oder die Temperatur kehrt im Anfall vorübergehend zur Norm zurück, sehen von $1 \frac{1}{2}$ Erythrozyten Durchmesser. Die anderen Autoren hatten, wie ich, völlig negative Resultate.

Der \%yklische Verlauf der Erkrankung macht die Annahme einer Protozoëninfektion jedenfalls am wahrscheinlichsten; möglicherweise kreisen diese nicht oder nur selten im strömenden Blut, sondern halten sich in den inneren Organen auf, vielleicht den langen Röhrenknochen, die meist der Sitz heftiger Schmerzen sind. Eine erhebliche Zerstörung von roten Blutkörperchen, wie bei Malaria, scheint nicht stattzufinden, da sich auch nach zahlreichen Anfällen keine stärkere Anämie ausbildet.

Zum Schluß komme ich noch auf die charakteristischen subjektiven Beschwerden unserer Kranken zu sprechen. Es sind dies heftigeKo pf- und Knochenschmerzen. Erstere sind vorwiegend in der Stirn, letztere in den Schienbeinen lokalisiert. Während der Intervalle waren sie mäßig, seltener hörten sie völlig auf; mit Beginn des Fiebers, manchmal schon einige Stunden früher, nahmen sie

um dann nochmals etwas anzusteigen (siehe Kurve 2, VII. Anfall). Einmal folgte noch eine mehrtägige Periode subfebriler Temperatur (Kurve 3).

Auf die kurzdauernden kleimen Temperatursteigerungen, die abortiven Anfällen entsprechen, habe ich schon oben hingewiesen.

Die Pulsfrequenz entspricht im allgemeinen der Temperaturhöhe.

Gemeinsam war allen meinen Fällen, daß sich an den inneren Organen nichts Krankhaftes nachweisen ließ, insbesondere war eine Milzschwellung weder palpatorisch noch perkutorisch nachweisbar, trotzdem täglich darauf hin unter- rasch an Heftigkeit zu und erreichten namentlich in der $\mathrm{Nacht}$ eine oft unerträgliche Stärke, sodaß oft völlige Schlaflosigkeit bestand. Meist gesellte sich dazu ein Gefühl quälender Unruhe in den Unterschenkeln, sodaß die Kranken sie hin- und herbewegen mußten („Auxietas tibiarum“). Einmal wurde über ähnliche Schmerzen in den Unterarmen geklagt, zweimal über Schmerzen in der linken vorderen Brustseite, ohne da $B$ hier etwas Krankhaftes nachweisbar war. Die Tibien waren bei einigen Kranken stark klopfempfindlich, namentlich an den

1) D. m. W. 1916 Nr. 15

.2) Nachtrag bei der Korrektur D. m. W. 1916 S. 411. 
Epiphysen. Die Nervenstämme waren niemals druckempfindlich. Störungen von seiten der Motilität, Sensibilität und der Reflexe waren nicht nachweisbar. Häufig hörten die Schmerzen mit dem völligen Sistieren der Fieberanfälle nicht auf, sondern bestanden noch wochenlang in wechselnder Intensität weiter, wobei manchmal die Steigerung der Beschwerden dem Füntageturnus entsprach. Besonders lang hielten sich die Kopfschmerzen. Auf diese Weise kamen manche Kranke recht herunter, und die Dienstfähigkeit war für längere Zeit aufgehoben.

In Uebereinstimmung mit den meisten anderen Autoren kann ich berichten, daß die üblichen Antipyretika (Aspirin, Pyramidon) und auch Chinin keinen Einfluß auf den Verlauf der Erkrankung hatten und auch die subjektiven Beschwerden nur in geringem Grade beeinflußten. In zwei Fällen gab ich 0,45 Neosalvarsan intravenös. In dem einen hatten die Fieberanfälle schon vorher aufgehört, aber die Schmerzen waren noch sehr quälend. Eine nennenswerte Besserung derselben' wurde mit der Einspritzung nicht erzielt. In dem anderen Falle folgte zwei Tage später wieder ein Anfall zu derselben Zeit; allerdings erreichte die Temperatur nicht ganz $38^{\circ}$, aber da es bereits der fünfte Anfall war, läßt sich hieraus nichts folgern. Die Schmerzen blieben auch bei diesem Kranken unbeeinflußt.

Ueber den Uebertragungsmodus der Krankheit kann ich auf Grund meiner Fälle kein Urteil abgeben. Bei den im Lazarett selbst Erkrankten ist die Uebertragung durch Läuse nicht wahrscheinhich.

Jedenfalls handelt es sich, wie meine Fälle zeigen, nicht um eine Erkrankung, die an eine bestimmte Gegend gebunden ist, sodaß die Bezeichnung "Wolhynisches Fieber" besser durch den Namen "Fünftagefieber" zu ersetzen ist. 